\title{
Chapter 6: Marine biological valuation of the shallow Belgian coastal zone: a space-use conflict example within the context of marine spatial planning
}

Sarah Vanden Eede, Lia Laporta, Klaas Deneudt, Eric Stienen, Sofie Derous, Steven Degraer, Magda Vincx

(in prep.) Marine biological valuation of the shallow Belgian coastal zone: a space-use conflict example within the context of marine spatial planning.

In preparation for submission to Ocean and Coastal Management 


\section{Abstract}

The Belgian coastal zone hosts a complex of space-use and resource-use activities with a myriad of pressures. Specifically at the beaches, predictions on sea-level rise, storms and flood risk from the North Sea have led to several big coastal defence projects. Management of sandy beaches is a multi-faceted and complex endeavor, where the interests of several stakeholders need to be combined.

In this paper, we used the marine biological valuation (BV) method in order to (1) analyse the spatial structure of the intertidal and shallow subtidal Belgian coastal zone; and (2) explore the applications of BV for an ecosystem-based approach to marine spatial planning of two space-use conflicts at the Belgian coast, being flood protection, by means of beach nourishment, and nature conservation.

The biological value was assessed with a focus on a detailed and integrated dataset (1995 - 2011), gathering all available ecological information on macrobenthos, epibenthos, hyperbenthos and birds. The $67 \mathrm{~km}$ Belgian coastline was divided into an across-shore intertidal and shallow subtidal subzone while the width of the along-shore subzones comprises $250 \mathrm{~m}$ for benthic components and wider distances of $3 \mathrm{~km}$ for the birds. The intrinsic biological value of each subzone was calculated using the BV method and the pertained score, ranging from very low to very high, was plotted accordingly in order to obtain a marine biological valuation map (BVM).

Following trends in BV along the Belgian coastline were detected: (1) a strong mosaic pattern of BV along the coastline; (2) a clear lack of (benthic) data at the eastern part of the Belgian coast; (3) a rather high biological value score for around $70 \%$ of the shallow part of the subzones, compared with the intertidal part; (4) a high/very high biological values found in intertidal zones located immediately to the east of the harbours Nieuwpoort, Oostende and Zeebrugge.

A detailed analysis of protected areas and areas under coastal flood risk indicates that the use of BVMs is very promising in order to differentiate between several impact values. BV can therefore be used as a management tool by local decision makers and can allow for the integration of 'natural/ecological values' at an early stage of policy implementation.

Key words: biological valuation, shallow coastal zone, space-use conflict, marine spatial planning 


\section{Introduction}

Marine and coastal waters are sensitive habitats that support high levels of biodiversity and provide many essential ecosystem goods and services (Costanza et al. 1998; de Groot et al. 2002; Beaumont et al. 2007; Beaumont et al. 2008). The escalating crisis in these ecosystems, from biodiversity losses and transformed food webs to marine pollution and warming waters, has been recognized to increasingly undermine the ocean's capacity of providing goods and services and maintaining resilience to stressors and changes (Worm et al. 2006). This crisis is in large part a failure of integrated governance (Crowder et al. 2006; Crowder \& Norse 2008). Current governance of marine systems is not place-based (Pikitch et al. 2004) but developed for particular marine resources and within individual economic sectors (Laffoley et al. 2004; Douvere 2008). In Belgium for instance, legal jurisdiction concerning coastal management is shared between the Flemish Government (landwards from the mean low water level (MLW)) and the Belgian State (seawards from the MLW). Such 'multi-level government' structure (Cliquet 2001, De Ruyck et al. 2001, Cliquet et al. 2007) most often results in conflicting priorities and overall lack of clarity in the implementation of relevant policies at the coastal zone (Commission of the European Communities 2007). It fails to provide a comprehensive integrated management of human activities, leading to fragmentation and spatial/temporal mismatches in governance. However, ecosystems, natural resources and human activities affecting coastal zones have place-based characteristics thus increasing the need to look at the 'system' from a spatial and temporal perspective. This also implies that all policies and management strategies (e.g. fisheries management, marine transportation management and marine protected area management) directed towards influencing human use of ecosystems and their resources, will inherently have a spatial and temporal dimension (McLeod 2005; Crowder \& Norse 2008).

During the last decade, marine spatial planning (MSP) has gained considerable importance in establishing ecosystem-based management in the marine environment. Ecosystem-based MSP seeks to attain not only consensus in sea-use management among distinct sectors, but also and most importantly to maintain the ecosystems' integrity and services through the conservation of marine biodiversity (Douvere 2008; Pomeroy \& Douvere 2008; Douvere \& Ehler 2009; Ehler \& Douvere 2009; Commission 2013b). This approach has been implemented in a few countries on a preliminary basis (Ehler 2008; Gilliland \& Laffoley 2008; Foley et al. 2010), including Belgium, although only in the marine offshore areas under federal jurisdiction. Biodiversity can be valued under several approaches and at several scales (Noss 1990; Oksanen 1997; Costanza 1999; Balvanera et al. 2006; Granek et al. 2009). In fact, the objectives behind each approach are directly linked with the respective definition of the term 'value' (Derous et al. 2007a). Most commonly, this is associated with the socio-economic value of ecosystems (Pearce \& Moran 1994; Costanza 1999), reflecting vestiges of the anthropocentric perspective over natural resources (Collet 2002). Valuing ecosystems by estimating the benefits they provide to society, accruing to ecosystems' goods and services, is an increasingly common practice in literature (de Groot et 
al. 2012). Under an ecosystem-based management approach however, biodiversity should also be valued intrinsically, independently of its potential usefulness for human beings (Wilson 1986; Ghilarov 2000).

The present work focuses on marine biological valuation (BV), a spatial tool that provides an integrated view on nature's intrinsic value, within a certain time frame (Derous et al. 2007a; Derous et al. 2007b). In this valuation method, all levels of biodiversity are assessed through a hierarchical ecological framework (Zacharias \& Roff 2000). By compiling all available biological and ecological information for a selected study area, and allocating an integrated intrinsic biological value to the subzones within the study area, biological valuation maps (BVMs) are produced. These maps facilitate the provision of a greater-thanusual degree of risk aversion in the management of activities as they are a tool for calling attention to areas which have particularly high ecological or biological significance (Derous et al. 2007a). Therefore, they can be used as reliable and meaningful baseline maps for spatial planning, marine policy and management approaches (Derous et al. 2007a; Derous et al. 2007b; Pascual et al. 2011). Hitherto, marine biological valuation has been performed in different European subtidal coastal waters (Derous et al. 2007d; Forero Parra 2007; Rego 2007; Vanden Eede 2007; Pascual et al. 2011) including the Belgian Part of the North Sea.

The goals of this paper are two-fold: (1) to analyse the ecological structure on a spatial scale of the intertidal and shallow subtidal Belgian coastal zone using the marine BV method; and (2) to explore the applications of BV for an ecosystem-based approach to MSP of two space-use conflicts at the Belgian coast, being flood protection, by means of beach nourishment, and nature conservation.

\section{Material and methods}

\subsection{Study Area}

The Belgian natural coastline (figure 1) is entirely composed of sandy beaches. The ecological continuum expected in this type of ecosystem, from the intertidal zone to the foredunes, is however disrupted by stone groynes and concrete dykes (De Ruyck et al. 2001), as a response to coastal flood risk (Speybroeck 2007; Roode et al. 2008). Previous research of the Belgian coastal ecosystem (Speybroeck et al. 2008a) suggested a zonation scheme, delimitating three main zones, along the tidal range: (i) the supralittoral zone, the area above the high water line influenced by sea water, represented by embryonic dunes, the dry beach area, and the drift line; (ii) the littoral or intertidal zone, the area comprised between high water and low water lines; and (iii) the infralittoral or shallow subtidal zone, represented by the subtidal foreshore as the seaward continuation of the beach profile until a depth of $4 \mathrm{~m}$ below the mean low water level (MLW). The subdivision of the shallow Belgian coastal zone follows this ecological zonation, focusing specifically on the intertidal and the shallow subtidal zones, and is defined by a landward 
boundary that follows the high water mark obtained by LIDAR observations of the Belgian coast in 2011 (data provided by the Agency for Maritime and Coastal Services: Coastal division - MDK) and a seaward boundary for the shallow subtidal foreshore of 1 nautical mile from the zero depth $(0 \mathrm{~m})$ bathymetric line (figure 1). The width of the subzones was chosen as fixed distances of $250 \mathrm{~m}$ for benthic components and wider distances (figure 1) of $3 \mathrm{~km}$ for birds, as these are highly mobile species (Derous et al. 2007c) .

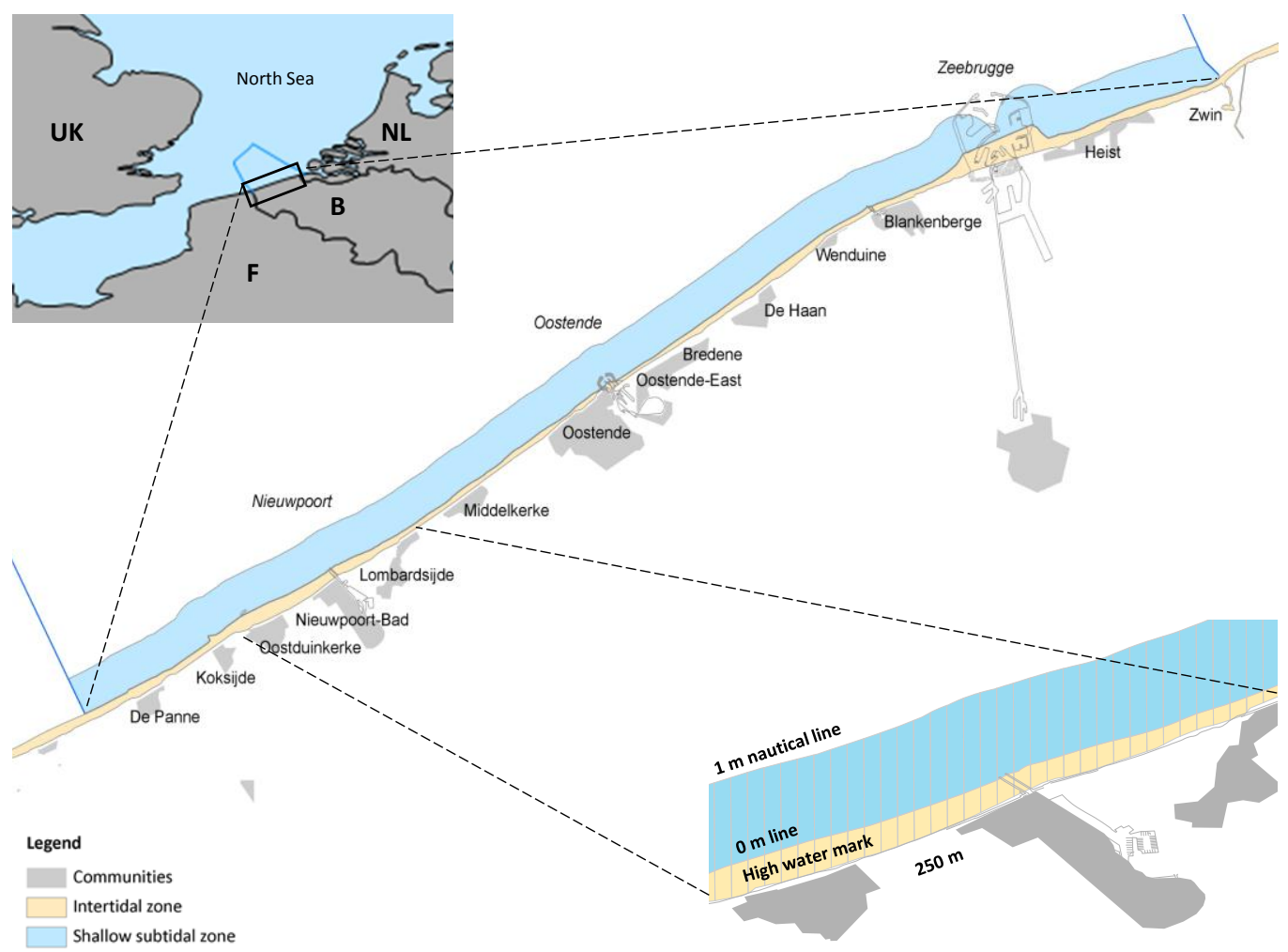

Figure 1: Study area of the Belgian coastal zone, with a distinction between the intertidal (light brown) and shallow subtidal zone (blue) and a detail showing the subdivisions performed for biological valuation

\subsection{Databases}

For the biological valuation of the shallow Belgian coastline, all available relevant data of benthos and birds in the intertidal and shallow subtidal zones during the period 1995-2011 were gathered (see Table 1 for references and sampling locations). The use of different sampling gears defines a differentiation among the benthic organisms: (i) macrobenthos - sampled with Van Veen grabs and/or quadrats and sieved over $1 \mathrm{~mm}$; (ii) epibenthos - sampled with $5 \mathrm{~mm}$ mesh size trawl nets (or push nets) over the bottom; and (iii) hyperbenthos - sampled with $1 \mathrm{~mm}$ mesh size trawl nets (or push nets), approximately $1 \mathrm{~m}$ above the bottom. The birds data were gathered through morning countings on the beach. The sampling strategy used for each ecosystem component was always the same. All datasets include the geographical coordinates, the sampling gear used and the area sampled. Species richness data (number 
of individuals per species and per sample) were standardized into densities (number of individuals per $m^{2}$.

Table 1: References used for the integrated database per ecosystem component. Restricted to data collected in intertidal and shallow subtidal zones of the Belgian coast (mainly from unpublished data of Marine Biology, Ghent University)

\begin{tabular}{|c|c|c|}
\hline Year of Collection & Sampling Locations & Reference \\
\hline \multicolumn{3}{|l|}{ MACROBENTHOS } \\
\hline 1995 & De Panne, Bray-Dunes and Koksijde & (De Neve 1996; Mouton 1996) \\
\hline 1997 & $\begin{array}{l}\text { De Panne ('De Westhoek'), Schipgatduinen, Koksijde, } \\
\text { Paelsteenpanne, ljzermonding, Lombardsijde, Raversijde, } \\
\text { Spinoladijk, Vosseslag, Blankenberge, Fonteintjes, } \\
\text { Zeebermduinen, Zeebrugge-bad, Baai van Heist, Heist, 'Zwin' } \\
\text { and VNR 'Zwinduinen en -polders' }\end{array}$ & $\begin{array}{l}\text { (Volckaert 1998; Speybroeck et al. } \\
\text { 2005b) }\end{array}$ \\
\hline 2001 & $\begin{array}{l}\text { Knokke-Heist, Blankenberge, Wenduine, Oostende, } \\
\text { Westende, Oostduinkerke, De Panne, Koksijde and } \\
\text { Zeebrugge }\end{array}$ & (De Backer 2001; Boulez 2002) \\
\hline $\begin{array}{l}2002,2004,2006 \\
2008,2009,2011\end{array}$ & $\begin{array}{l}\text { Lombardsijde, Nieuwpoort, Bredene, Koksijde- } \\
\text { Oostduinkerke, Oostende (Centrum, Oosteroever, Vaargeul), } \\
\text { Wenduine, Blankenberge, Mariakerke }\end{array}$ & Beach Nourishment Project ${ }^{1}$ \\
\hline \multicolumn{3}{|l|}{ EPIBENTHOS } \\
\hline 2001 & Koksijde & (Buyle 2002) \\
\hline 2003 & $\begin{array}{l}\text { De Panne ('De Westhoek'), ljzermonding, VNR 'Zwinduinen } \\
\text { en -polders', Spinoladijk, Fonteintjes, Raversjide, } \\
\text { Zeebermduinen, Schipgatduinen, Zeebrugge-bad, Baai van } \\
\text { Heist, Paelsteenpanne }\end{array}$ & $\begin{array}{l}\text { (Speybroeck et al. 2005b) } \\
\text { (Speybroeck et al. 2005b) }\end{array}$ \\
\hline \multicolumn{3}{|l|}{ HYPERBENTHOS } \\
\hline 1997 & Lombardsijde & (D'Hondt 1999) \\
\hline 2001 & Koksijde & (Buyle 2002) \\
\hline 2003 & $\begin{array}{l}\text { De Panne ('De Westhoek'), ljzermonding, VNR 'Zwinduinen } \\
\text { en -polders', Spinoladijk, Fonteintjes, Raversjide, } \\
\text { Zeebermduinen, Schipgatduinen, Zeebrugge-bad, Baai van } \\
\text { Heist, Paelsteenpanne }\end{array}$ & (Speybroeck et al. 2005b) \\
\hline \multicolumn{3}{|l|}{ BIRDS } \\
\hline 2003 and 2004 & $\begin{array}{l}\text { De Panne ('De Westhoek'), ljzermonding, VNR 'Zwinduinen } \\
\text { en -polders', Fonteintjes, Raversjide, Zeebermduinen, } \\
\text { Schipgatduinen, Zeebrugge-bad, Baai van Heist, } \\
\text { Paelsteenpanne }\end{array}$ & (Speybroeck et al. 2005b) \\
\hline
\end{tabular}

\footnotetext{
${ }^{1}$ Beach nourishment project: Speybroeck et al. 2003, Welvaert 2005, Van Ginderdeuren et al. 2007, Vanden Eede et al. 2008, Vanden Eede \& Vincx 2010, 2011, 2013
} 


\subsection{Biological Valuation Protocol}

\section{Method application}

The purpose of marine biological valuation is to provide an integrated view on nature's intrinsic nonanthropogenic value of the subzones (but relative to each other), within a study area (Derous et al. 2007d). Unlike the previous applications of the protocol (Derous et al. 2007c; Forero Parra 2007; Rego 2007; Vanden Eede 2007; Weslawski et al. 2009; Pascual et al. 2011), the procedure used now is effectuated based on $R$, which is open-source software for statistical computing and graphics ${ }^{2}$. The $R$ script for marine biological valuation has been recently developed by the Flanders Marine Institute (VLIZ), in Oostende, Belgium (Deneudt et al., submitted). Due to the fact that the protocol is flexible and subject to specific adaptations for each application, each of the steps, used for this valuation of the Belgian beaches, will be explained in the following subsections.

The $R$ script for marine biological valuation guarantees general data quality control (geographical coordinates, dates, time and taxonomy, based on the World Register of Marine Species (WoRMS ${ }^{23}$ ). The set of assessment questions (table 2 ) relates the available biological data to the valuation criteria, being rarity and aggregation-fitness consequences, and to a specific organizational level of biodiversity. These valuation criteria were proposed by Derous (2007), after an extensive literature review and selection based in part on the framework for identification of Ecologically Significant and Biologically Significant Areas (DFO 2004) and expert judgment (Derous et al. 2007c). Biodiversity is not included as a separate valuation criterion, but linked to one or more of the selected valuation criteria using the 'marine ecological framework' created by Zacharias and Roff (2000).

Table 2: Set of assessment questions (Derous et al. 2007c)

\begin{tabular}{ll}
\hline Assessment Question & Categories of Species \\
\hline Is the subzone characterized by high counts of many species? & all species \\
Is the abundance of a certain species very high in the subzone? & all species \\
Is the abundance of rare species high in the subzone? & rare species \\
Is the subzone characterized by the presence of many rare species? & rare species \\
Is the species richness in the subzone high? & all species \\
Is the abundance of ecologically significant species high in the subzone? & ecologically significant species \\
Is the abundance habitat-forming species high in the subzone? & habitat-forming species \\
\hline
\end{tabular}

The assessment questions are based on several 'categories of species', such as all species, rare species, ecologically significant species and habitat-forming species (table 2) giving differential value to some

\footnotetext{
2 http://www.r-project.org/

3 http://www.marinespecies.org/
} 
species categories. For all species, species richness is calculated as the mean species richness per sample, location and subzone. Some sensibility to sampling effort bias cannot be excluded when using this calculation but it remains limited as the sampling method is uniform per ecosystem component and the species richness is calculated per sample. Derous et al. (2007) determined the criteria on rare species, by their percentage of occurrence in the samples: rare species were defined as those appearing in less than $5 \%$ of the studied subzones. However, this threshold can be changed if properly justified as is the case when all species occur in more than $5 \%$ of the subzones and as such no rare species can be determined. Since the protocol was designed to be flexible and aims at offsetting the relative differences between subzones as much as possible, the threshold was elevated to $10 \%$. Therefore, rare species were defined as those appearing in less than $10 \%$ of the studied subzones. Habitat-forming species (HFS) were selected based on expert judgment, supported by the extensive literature existent on the role of such species dwelling the Belgian coast and continental shelf (Hiittel 1990; Rasmussen et al. 1998; Callaway 2006; Rabaut et al. 2007; Van Hoey et al. 2006; Rabaut 2011). Ecologically significant species (ESS) were selected based on expert judgment assessment and literature review (Van Hoey et al. 2005, 2007). It should be noted that subjectivity cannot be totally excluded in this BV method. A list of selected HFS and ESS, and the rationale behind this selection can be found in Appendices - Chapter 6 - Appendix A.

The assessment questions for each of the ecosystem components need to be translated into mathematical algorithms (see Appendices - Chapter 6 - Appendix B). Solving these algorithms yields a numeric answer to each assessment question, corresponding to a score translated into a semiquantitative classification system of five value classes: very low, low, medium, high and very high BV. If there is no data to answer a specific question for a certain subzone, this is labeled ' $N A$ '. An example of the scoring process described above can be seen in Appendices - Chapter 6 - Appendix C1. The scores for all assessment questions are added together per subzone, though separated for different ecosystem components and bearing in mind that each assessment question has been attributed an equal weight in the total score. These results are then illustrated in a BVM per ecosystem component.

The reliability of the assessed values for each subzone are noted with an attached label, perceptible in the final map (low, medium, high). Such label can either display the amount and quality of the data used to assess the value of a certain subzone (data availability) or it displays how many assessment questions could be answered per subzone given data availability (reliability of information). For example, when a certain question cannot be answered for one or more subzones, these subzones are scored on the basis of the remaining questions (the ones that could be answered), decreasing the completeness of the information and the reliability of the scoring. On a further level, when certain subzones lack data for one or more ecosystem components, these are valued based on the final score for the remaining available ecosystem components only, being less reliable than subzones valued based on all of the ecosystem components. An example of how data availability and reliability of information have been incorporated 
into the protocol can be seen in Appendices - Chapter 6 - Appendix C. These reliability labels and the BVMs should be consulted simultaneously as they allow us to identify knowledge gaps.

The total biological value of the subzones is determined by averaging the intermediate values for the different ecosystem components. An example of how to perform the final scoring can be seen in Appendices - Chapter 6-Appendix C4. The results of the BV are then presented on a final BVM, where each subzone is assigned a color corresponding to its resulting biological value. Both reliability and availability labels of each subzone are displayed on the BVM by using different intensities of color or different fillings.

\section{Using BV for solving space-use conflicts, e.g. flood risk and nature conservation}

After a final BVM map of the Belgian coastal zone was obtained, the applications of this map were investigated. For the flood risk scenario, information regarding areas already identified as extremely vulnerable to coastal flood risk, and hence highly likely of undergoing coastal defence activities in the near future, has been collected and transformed into a spatial layer for analysis (see Appendices Chapter 6 - Appendix D1). The final BVM was displayed along with this spatial layer. In order to analyse the results from a management perspective, spatial data joining was performed using the final BVM and the ten delimited Belgian coastal areas covered by Provincial Spatial Implementation Plans (PSIPs) (Appendices - Chapter 6-Appendix D1).

For the nature conservation scenario, the final BVM was displayed together with the existing protected areas at the shallow Belgian coastal zone, under European (RAMSAR, Birds \& Habitat Directive combined in the Natura 2000 Network - Special Areas of Conservation \& Special Protection Areas) and National/Flemish legislation (marine/nature reserves, and protected dunes) (see Appendices - Chapter 6 - Appendix D2). Data were obtained from the interactive coastal atlas of the Flemish Region (Maelfait \& Belpaeme 2009).

\section{Results}

\subsection{BVM per ecosystem component}

The BVMs for birds, macrobenthos, epibenthos, and hyperbenthos can be seen in Appendices - Chapter 6 - Appendix E1, E2, E3 and E4 respectively. The reliability indices, data availability and information reliability, per ecosystem component are depicted in the maps of Appendices - Chapter 6 - Appendix F1, F2, F3 and F4. Information reliability was maximal (high) for all subzones with data, meaning the chosen assessment questions for each ecosystem component could be answered in every subzone with data. Table 3 shows the number of subzones with data per ecosystem component. It is clear that the 
ecosystem component 'macrobenthos' delivers the highest amount of data for the total valuation. To check whether data availability is correlated with the valuation scores, a simple Pearson correlation was performed (table 3). No correlation could be detected.

Table 3: Number and percentage (\%) of subzones with data, out of the total number of subzones per ecosystem component; Pearson correlation $(r)$, with corresponding coefficient of determination $\left(r^{2}\right)$ between data availability and BV scores per ecosystem component

\begin{tabular}{lcccc}
\hline Ecosystem component & Total number of subzones & Number of subzones with data (\%) & $r$ & $r^{2}(\%)$ \\
\hline Birds & 42 & $10(24 \%)$ & 0.30 & $0,09(9 \%)$ \\
Macrobenthos & 463 & $124(27 \%)$ & -0.40 & $0,16(16 \%)$ \\
Epibenthos & 463 & $11(2 \%)$ & 0.73 & $0,53(53 \%)$ \\
Hyperbenthos & 463 & $14(3 \%)$ & 0.16 & $0,03(3 \%)$ \\
Total valuation & 463 & $216(47 \%)$ & 0.21 & $0,04(4 \%)$ \\
\hline
\end{tabular}

\subsection{Integrated BVM}

Figure 2 shows the final BVM for the Belgian coastal zone. The mosaic-like variability of scores is apparent and can also be seen in the BVM of macrobenthos (Appendices - Chapter 6 - Appendix E2). There is a clear difference in the amount of data collected to the west of Oostende if compared to the east and around $70 \%$ of the shallow subtidal subzones with data scored medium, high or very high. Moreover, biologically high valued intertidal zones are not necessarily bordered by biologically high valued shallow subtidal zones and vice versa. Both final reliability indices, information reliability and data availability, are mapped together in figure 3 . Most subzones displayed medium to high information reliability and have a low or medium data availability. High/very high biological values are consistently found in intertidal zones located immediately to the east of the three prominent Belgian harbours (figure 4). 
Chapter 6 - Marine biological valuation of the shallow Belgian coastal zone: a space-use conflict example within the context of marine spatial planning

Legend

Communities

Marine Biological Valuation

NA

Very Low (1)

Low (2)

Medium (3)

High (4)

Very High (5)

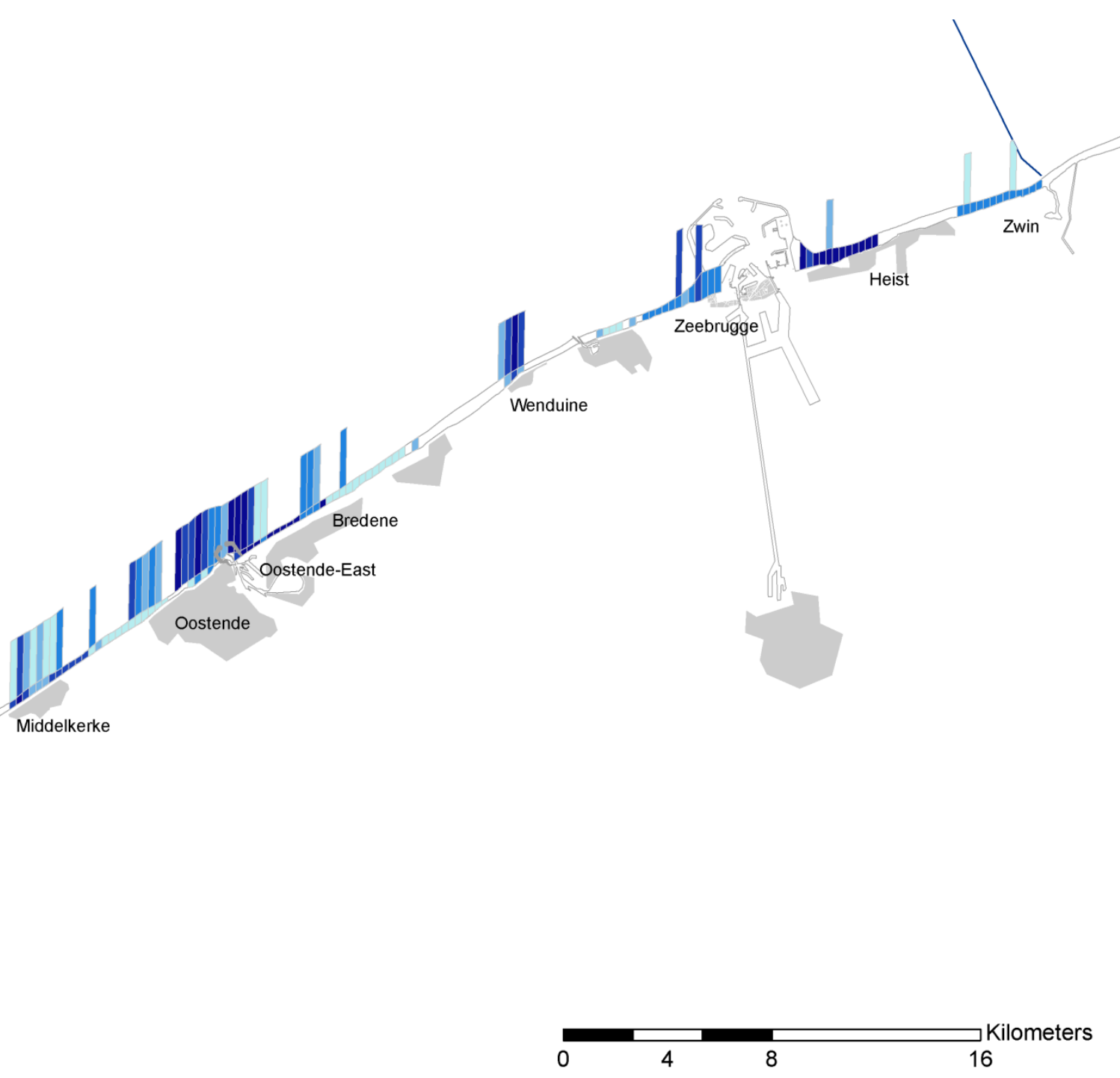

Figure 2: Final BVM for the Belgian coast 
Chapter 6-Marine biological valuation of the shallow Belgian coastal zone: a space-use conflict example within the context of marine spatial planning

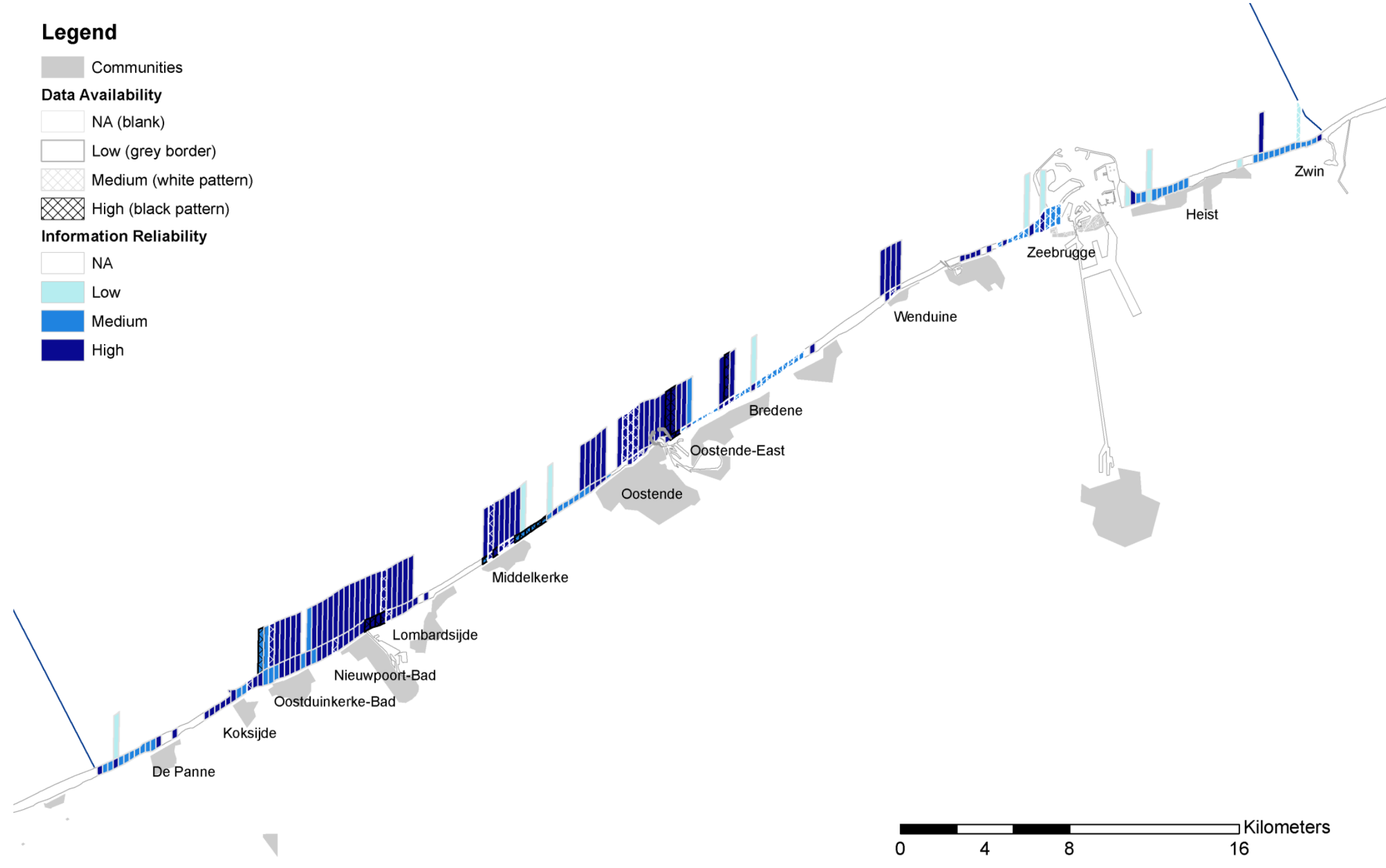

Figure 3: Final map depicting Information Reliability and Data Availability for the Belgian coast 


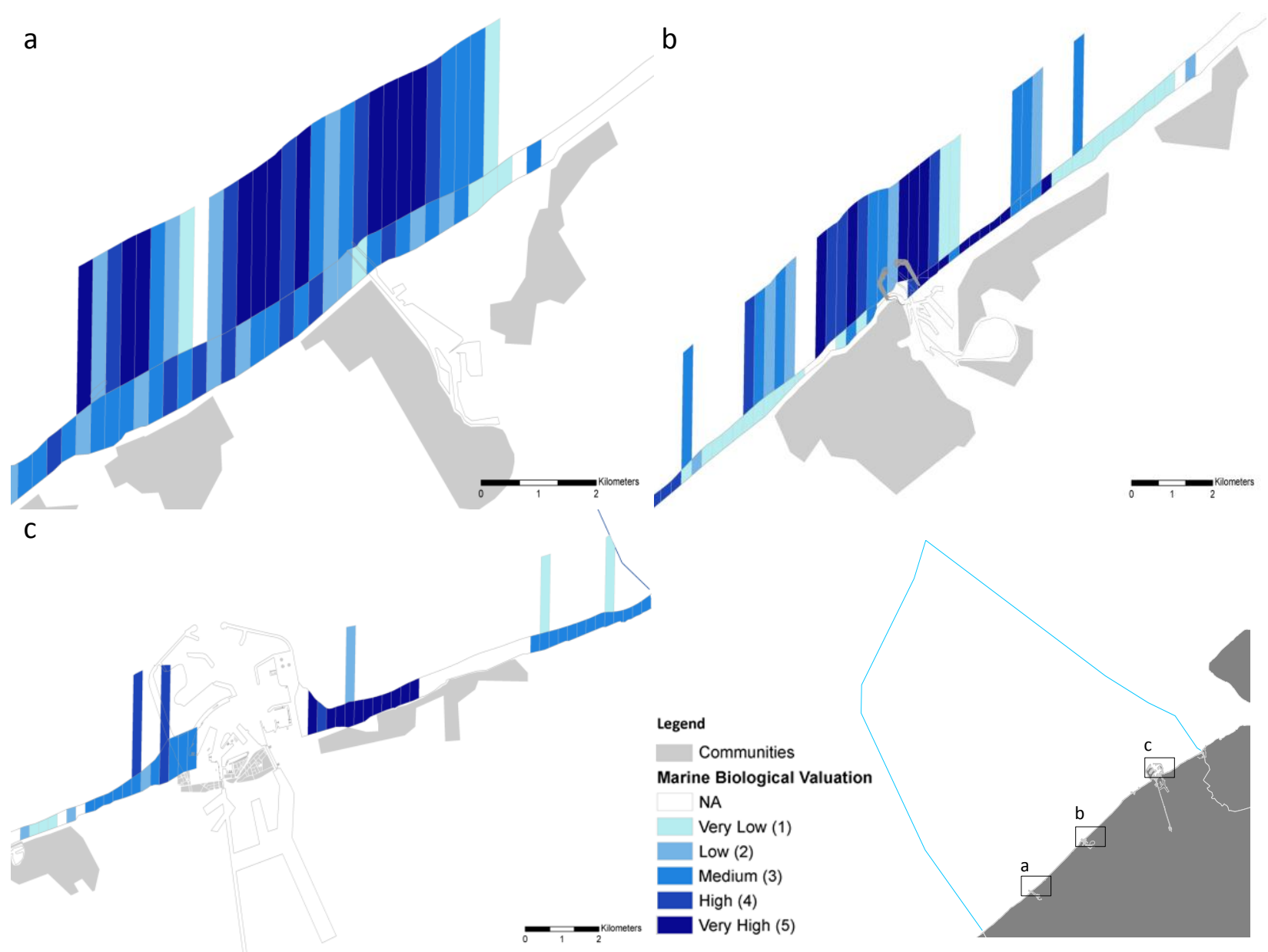

Figure 4: Detailed information on the BV of areas located at the east side of the main harbours at the Belgian coast:

(a) Nieuwpoort (Lombardsijde); (b) Oostende (Oostende-East); (c) Zeebrugge (Baai van Heist).

\subsection{Using BV for solving space-use conflicts}

The final BVM was displayed along with areas under coastal flood risk (Appendices - Chapter 6 Appendix D1) and along with the PSIPs. Since the PSIPs only cover the intertidal part of the Belgian beaches, the maps in figure 5 and Appendices - Chapter 6 - Appendix $G$ only show the biological value of the intertidal area. Figure 5 focuses on the harbour areas as they have been given high coastal defence priority in the current Integrated Master Plan for the Flemish coast (Mertens et al. 2008) and the areas just east of the harbours seem to attain a high/very high biological value (figure 4). Areas for which no spatial plan exists, e.g. the beach of Lombardsijde, are commonly addressed as blank or undesignated areas (figure 5a) (Bogaert \& Maes 2008). Areas sensible to coastal flood (in red) but lacking biological data (no color) were identified within almost all of the PSIPs (figure 5c). Areas sensible to coastal flood and displaying high/very high biological value were also identified (figure $5 \mathrm{a}$ and $5 \mathrm{c}$ and Appendices Chapter 6-Appendix G1 to G6). 


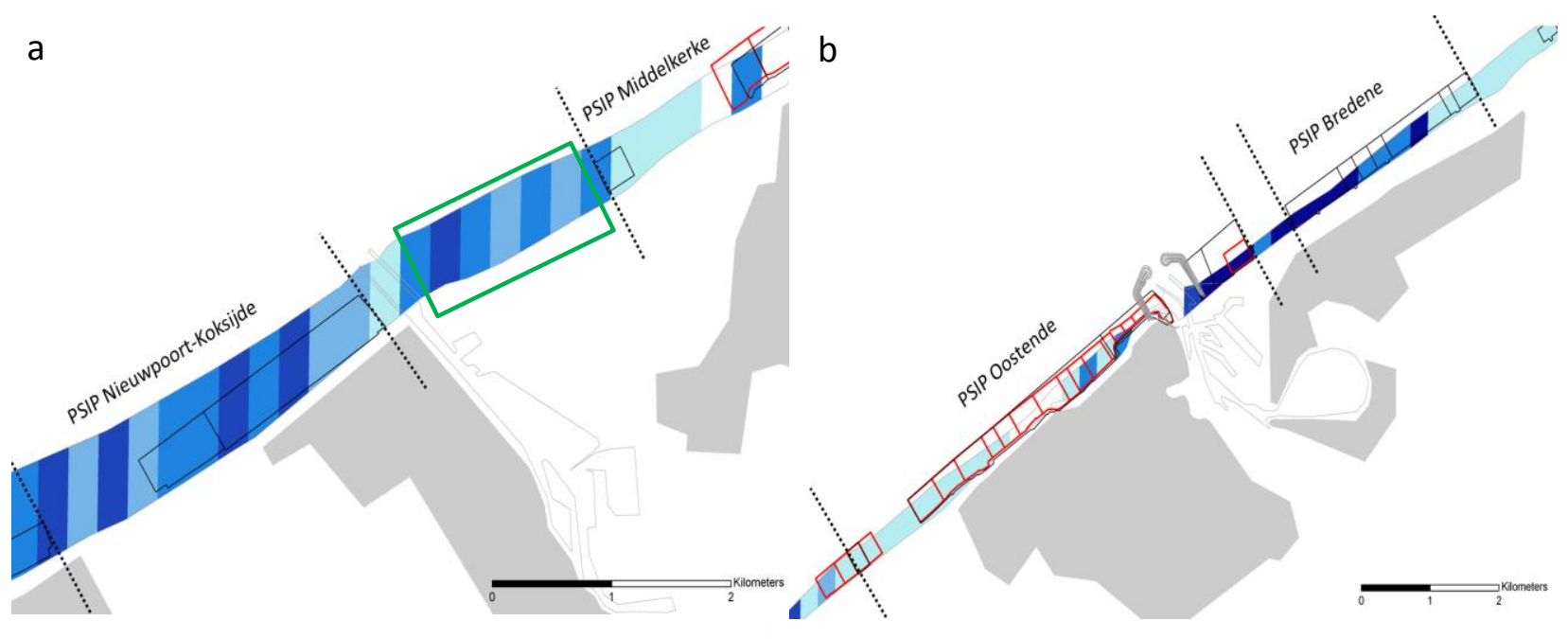

C
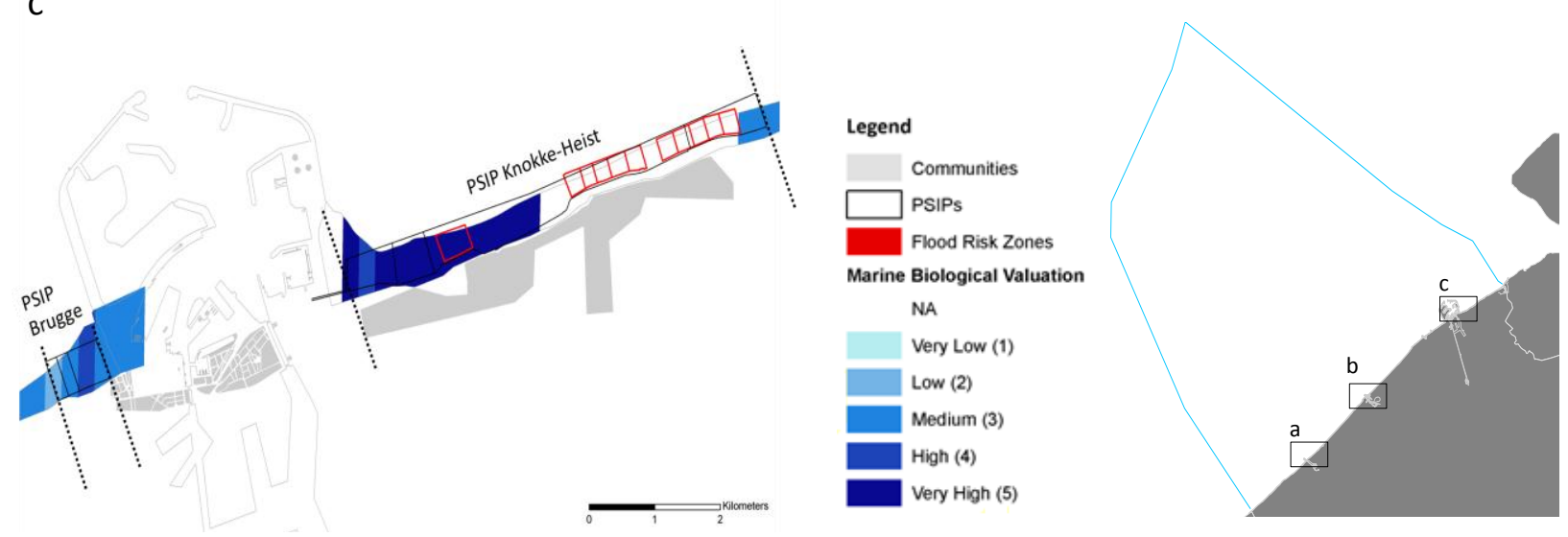

Figure 5: Detailed map with BV scores of intertidal areas located at the east side of the main harbours at the Belgian coast, inside PSIPs. Red indicates areas under coastal flood risk. The dashed lines mark the boundaries of each PSIP: (a) Nieuwpoort (Lombardsijde); the beach of Lombardsijde (green rectangle) falls inside an undesignated area as it is not covered by any PSIP (Maes \& Bogaert 2008); (b) Oostende (Oostende-East); (c) Zeebrugge (Baai van Heist);

Considering the nature conservation scenario, all protected areas in the shallow Belgian coastal zone are displayed together with the final BVM (Appendices - Chapter 6 - Appendix D2). Detailed maps of the most important protected areas are shown in figure 6. Overall low BV scores for De Panne and 'De Westhoek' (figure 6a) and the medium intertidal value and low shallow subtidal value for 'Zwin' (Figure 6c) were certainly lower than expected. Lombardsijde beach area of the Flemish nature reserve 'IJzermonding' gets a medium/high intertidal score and a very high shallow subtidal score (figure 6b). The Flemish nature reserve 'Baai van Heist' (figure 6c) attained a very high BV. 


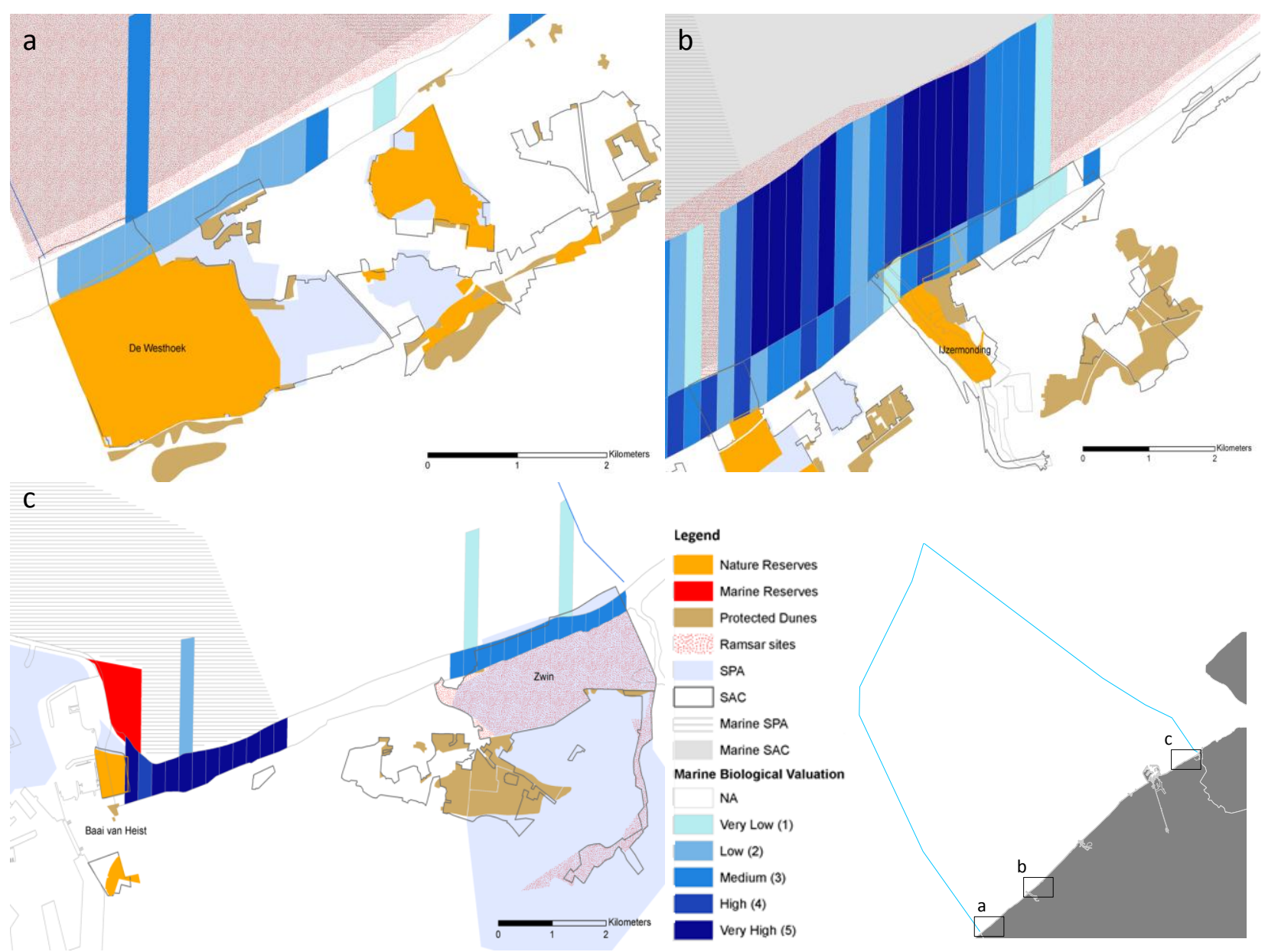

Figure 6: Detailed information on the BVM of protected areas located at the Belgian coast:

a) 'De Westhoek' (De Panne): only low intertidal scores were obtained despite its ecological importance;

b) Nature Reserve IJzermonding (Lombardsijde): very high valuation scores were obtained for the shallow subtidal waters adjacent to Lombardsijde beach, providing a visual support for the extension of the reserve seawards;

c) Zwin: an overall medium score, whereas intertidal subzones located near Baai van Heist have high/very high scores

\section{Discussion}

\subsection{Integrated BVM of the Belgian coast}

According to table 3 , data used in this biological valuation covers almost half of the total study area (47\%), with the ecosystem component 'macrobenthos' delivering the highest amount of data for the total valuation. A simple correlation test was performed in order to check if the amount of data obtained in each subzone would be influencing the valuation score (table 3). Although a relatively higher $r^{2}$ was obtained for epibenthos (0.53), overall $r^{2}$ values were low and showed no strong correlation between the variables. The datasets used for epibenthos and hyperbenthos have been incorporated into the final 
valuation although they cover only around $3 \%$ of the study area each (table 3), not allowing to deliver reliable results on these two ecosystem components as data availability and spatial coverage are just too far from satisfactory (Appendices - Chapter 6-Appendix E3 and F3; E4 and F4 respectively). Table 3 also shows that the macrobenthos dataset is definitely the largest and as such contains data for the majority of the subzones. Most observed trends of the integrated BVM can be explained by taking a closer look at the BVM of macrobenthos (Appendices - Chapter 6-Appendix E2).

Firstly, the mosaic-like variability of scores is apparent in both the final BVM (figure 2) as well as in the BVM of macrobenthos (Appendices - Chapter 6 - Appendix E2). This can be explained by the irregular and patchy distribution of sediments in the coastal zone due to minor across-shore and along-shore morphodynamic and morphological differences (Degraer et al. 2003b; Van Hoey et al. 2004; Vanden Eede et al. 2013, in prep.). Combined with the diverse topography of the Belgian coastal zone, this creates a wealth in habitats supporting a high capacity for varied benthic species assemblages (Van Hoey et al. 2004). Secondly, there is a clear difference in the amount of data collected to the west of Oostende if compared to the east. Furthermore, information at the eastern part of the Belgian coast is much scarcer, even for areas of great ecological importance such as 'Baai van Heist' or 'Zwin'. This is easily explained since the largest clusters of data (Lombardsijde, Nieuwpoort, Bredene, KoksijdeOostduinkerke, and Oostende) were gathered during sampling campaigns in the framework of environmental assessments for beach nourishment projects, which are located mostly westwards of Oostende. Thirdly, around $70 \%$ of the shallow subtidal subzones with data scored medium, high or very high. The breakdown of this result shows that these high values were obtained through questions related to Aggregation-Fitness consequences. Due to specific abiotic conditions, species richness and abundance of benthic organisms (Dewicke et al. 1998), shallow Belgian coastal waters are indeed known as nursery areas for a series of epibenthic macro-crustaceans and flatfish species (Rabaut et al. 2010). Nevertheless, for the question on ESS, higher values are mostly found in the shallow subtidal, suggesting that the ESS selected (Appendices - Chapter 6-Appendix A) are perhaps not equally capturing intertidal and shallow subtidal communities. For example, although the Abra alba community is extremely important in subtidal waters (Van Hoey et al. 2005, 2007a), the emphasis given to this species by naming it an ESS might have caused an underestimation of the overall ESS scores for intertidal subzones. Finally, a mismatch between the intertidal and shallow subtidal scores can be detected. Biologically high valued intertidal zones are not necessarily bordered by biologically high valued shallow subtidal zones and vice versa. Although there seems to be a gradual transition in macrobenthic assemblages from the lower intertidal to the shallow subtidal zone (Defeo \& McLachlan 2005; Speybroeck et al. 2008a), the differences in these assemblages between both zones are substantial enough to lead to different scores by applying the same assessment questions.

Reliability of information apprises the level of certainty of the obtained BV scores, whereas data availability pinpoints subzones with more or less sampling effort, indicating where future surveys should 
be undertaken (Pascual et al. 2011). Hence, increasing reliability and sampling effort leads to a higher level of certainty of the final BV scores. The assessment questions chosen aimed at addressing the type of data integrated in this valuation. Most subzones displayed medium to high information reliability and have a low or medium data availability (Figure 3).

\subsection{Using BV for solving space-use conflicts}

\section{Coastal defence}

In addition to the trends previously discussed, another important pattern has been observed. High/very high biological values are consistently found in intertidal zones located immediately to the east of the three prominent Belgian harbours (figure 4). The major wind-driven and tidal currents and waves at the Belgian coast have a southwest-northwest direction (van der Molen \& van Dijck 2000; Speybroeck et al. 2008a). As a consequence of the net sediment transport towards the northeast, current-induced erosion causes depletion of sediments to the west of these hard structures and sediment deposition at the east side, in a kinematic process already described and commonly addressed in coastal geophysics (Deronde et al. 2004). The east side of these prominent hard structures (also referred to as lee-side) is a sheltered area where hydrodynamics are less intense and sand deposition occurs. Hence, it creates a wealth in soft bottom habitats and proper environmental conditions for benthic colonization, which goes in accordance with the observed pattern.

The spatial correlation between the final BVM and the PSIPs (Appendices - Chapter 6 - Appendix G1 to G6) showed that areas for which no spatial plan exists are commonly addressed as blank or undesignated areas (Bogaert \& Maes 2008) and as such cannot be legally considered under the scope of coastal spatial management. Lombardsijde beach, part of the nature reserve 'IJzermonding' (Figure $5 \mathrm{a}$ and 6b), is such an undesignated area but its high/very high BV scores emphasize the importance of a full-coverage coastal network of PSIPs, leaving no room for undesignated areas. Areas sensible to coastal flood (in red) but lacking biological data (no color) are identified within almost all of the PSIPs, e.g. the beach zone between Knokke-Heist and Zwin (figure $5 \mathrm{c}$ ). Areas sensible to coastal flood and displaying high/very high biological value are also identified (Appendices - Chapter 6 - Appendix G1 to G6), e.g. Middelkerke (figure 5a), Oostende Oosteroever (figure $5 b$ ) and Knokke-Heist (figure $5 c$ ). If coastal defence activities are to be performed in these areas, appropriate (mitigation) measures have to be drafted. This stresses the need for acquiring more relevant biological data at the unstudied areas with high coastal flood risk. Some critical steps for an ecologically good practice of beach nourishment should be taken, particularly in areas of high/very high BV, such as: (1) selection of nourishment techniques in respect to local natural values; (2) selection of sand nourishment based on the sediment composition of the targeted area (grain size); (3) avoiding drastic alteration of the beach slope; (4) execution of nourishment activities during periods of low beach activity of birds or other mobile organisms; and (5) favoring the selection of 
smaller, phased projects as opposed to a single, wide project (Peterson et al. 2000; Speybroeck et al. 2006a).

An alternative nourishment solution, known as foreshore nourishment, involves the implementation of parallel sandbanks along the entire coast just at the submerged foreshore. These sandbanks constantly supply sand to the beach zone after progressive tidal regimes (Misdorp \& Terwindt 1997). However, intertidal communities are much more adapted to extreme sudden changes in environmental conditions than subtidal ones (Speybroeck et al. 2005a), making them relatively more resilient to anthropogenic interventions such as beach nourishment. Additionally, habitat continuity from the low intertidal zone to the foreshore (Degraer et al. 1999a) is disrupted by these sandbanks, hindering repopulation of the low intertidal zone by subtidal organisms. The high/very high BV obtained for most shallow subtidal zones along the Belgian coast (figure 2) further stress the need for caution when contemplating coastal defence measures such as foreshore nourishment. Overall, it can be concluded by saying that these results highlight the potential usefulness of BVMs for coastal and marine spatial planning in Belgium, particularly if considered as baseline maps under a solid decision support system (figure 7).

\section{Nature Conservation}

The BV protocol has achieved good results as a tool for the implementation of the Habitats and Birds Directives in the Belgian Part of the North Sea (Derous et al. 2007d) and as a framework in the environmental status assessment, under the European Marine Strategy Framework Directive (Borja et al. 2011; Pascual et al. 2011). It could also be used as a baseline map for the implementation of the European Water Framework Directive, as the protocol incorporates most of the biological and physical characteristics required by the Directive (Derous et al. 2007d). To stress the usefulness of the BV protocol as support tool for the proposal of new or the extension of already existing protected areas, the integrated coastal BVM was displayed along with the main protected areas at the Belgian coast (Appendices - Chapter 6 - Appendix D2). It is clear that not all areas with a kind of protection status, have a high ecological value, as defined with the BV method. This can be due to several reasons, as explained below.

For the area of De Panne, both the birds and macrobenthos BVM show a low BV (Appendices - Chapter 6 - Appendix E1 and E2) leading to overall low BV scores (figure 6a). Nevertheless, the ecological importance of De Panne and the grey dunes of 'De Westhoek' have been widely acknowledged in literature (Bonte et al. 2004; Provoost et al. 2004; Vandenbohede \& Lebbe 2004) and the latter is even a reserve considered to be properly managed from an ecological perspective (De Ruyck et al. 2001; Houston 2003). However, literature also allocates the ecological importance of both areas to the ecosystem components vascular plants and terrestrial arthropods. Since there was insufficient data for these components, they were not included in this analysis. As such, no significant conclusions regarding 
the biological value of De Panne and 'De Westhoek' can be made due to the lack of information on vascular plants and insects, the sparse distribution of subzones with data and the absence of shallow subtidal information.

High data availability in the Lombardsijde beach area of the Flemish nature reserve 'IJzermonding' supports a medium/high intertidal score and a very high shallow subtidal score (figure 6b). However, the beach of Lombardsijde is an undesignated area on the PSIPs since it falls under military jurisdiction. It was proposed for special management plans in $\mathbf{2 0 0 0}$ given its high ecological importance (Herrier \& Van Nieuwenhuyse 2005). The very high shallow subtidal scores of Lombardsijde beach justify and underline the ecological importance of extending the beach reserve seawards (figure $6 \mathrm{~b}$ ) by providing a straightforward and visual message to support this advice (Van Nieuwenhuyse 2003).

The Flemish nature reserve 'Baai van Heist' (figure $6 \mathrm{c}$ ) attained a very high BV due to the birds' valuation. This was expected, as the development of the harbour of Zeebrugge in the 1980s created vast areas of sandy, sparsely vegetated and relatively undisturbed coastal areas, mimicking natural processes and attracting a great number of coastal breeders (Stienen \& Van Waeyenberge 2002; Stienen \& Van Waeyenberge 2004; Stienen et al. 2005). In fact, the distribution of species such as Sterna albifrons (Little tern) is now almost exclusively limited to this area and adjacent beaches (Courtens \& Stienen 2004; Stienen et al. 2005).

Protected under various legislations and directives, 'Zwin' is one of the most important protected areas of the Belgian coast (figure $6 \mathrm{c}$ ). Its ecological relevance is related not only to its role as a breeding/feeding/aggregation site for birds but also to the presence of rare and important species (Devos 2008; Herrier \& Leten 2010; Charlier 2011; BirdLife 2013). The medium value obtained for 'Zwin' (Figure 6c) was certainly lower than expected. The value is strongly influenced by the results for the birds, suggesting that the birds' data are not covering the real situation. The low score for the shallow subtidal subzone of 'Zwin' (Figure 6c) was only valued on the basis of epibenthos and hyperbenthos, scoring very low and low, respectively. Although little can be discussed for these components separately, previous literature suggested a decline of species richness and abundance for hyperbenthic communities under estuarine influence (Dewicke et al. 2003). Being in such proximity to the Scheldt estuary, this might very well be the case for 'Zwin' but without a better spatial coverage of data, this remains a mere speculative conclusion.

Clearly, more comprehensive datasets need to be incorporated in future biological valuations of the Belgian coast, particularly for the beach of De Panne and the 'Zwin' area. 


\section{$B V$ as tool for ecosystem based-marine spatial planning at the Belgian coast}

Ecosystem-based definitions and strategies should only be used if they are able to inform management actions based on an intrinsic assessment of biological value (Arkema et al. 2006). BV can be a valuable tool within the scope of EB-MSP at the Belgian coast as it allows for the integration of 'nature' at an early stage of policy implementation, for both coastal flood risk and nature conservation space-use conflicts. The BVMs permit informing management decisions at a level that is closer to stakeholders, significantly attenuating conflicts and enabling a transparent involvement (Pomeroy \& Douvere 2008; Fleming \& Jones 2012). Still, BVMs should be further considered together with other criteria related to socio-economic and political/legal preconditions within an integrative decision support system for spatial planning (Derous et al. 2007c)(figure 7).

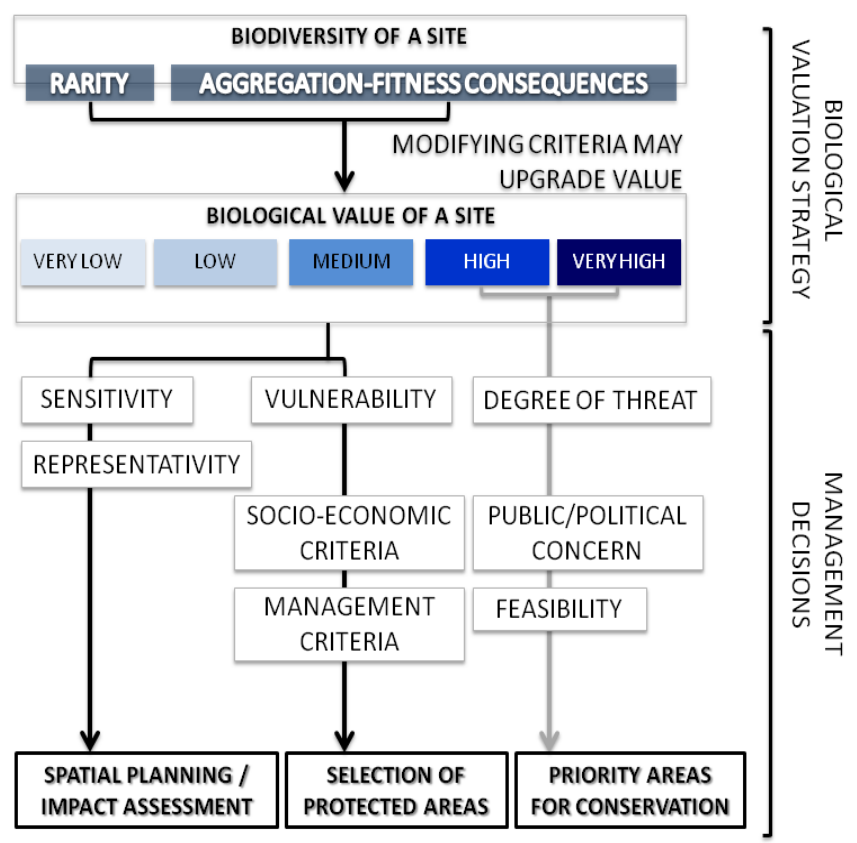

Figure 7: Overview of the BV concept and possible future steps to develop decision support management approaches (adapted from Derous et al 2007c)

\section{Positive aspects of BV}

When valuing marine biodiversity, it is important to capture as many attributes of biodiversity as possible, since biological structures and processes exist on different organizational levels (Zacharias \& Roff 2000). Even though in this work the data available only addresses biological structures at the species/population and community levels, larger and more comprehensive datasets would eventually allow for the incorporation of all levels of biodiversity. Furthermore, the BV protocol also allows for the formulation and selection of different assessment questions, based on the ecological knowledge of the study area, and the inclusion of data regarding biological processes and functions (e.g. the presence of migratory routes and upwelling sites or overall productivity of a subzone), leading to more ecologically meaningful results.

BVMs only have a medium-term reliability and should be updated after a relevant period of time (several years) to reflect the medium-term variability in biological value and to meet the dynamics of the marine and coastal ecosystem. Unfortunately, the necessary high sampling intensity restrains a frequent update of BVMs making it impossible to reflect real inter-seasonal or inter-annual differences in biological value. For the time being, only maps based on data from a longer time period, giving a summary of the medium-term variability in value, can be developed (Derous et al. $2007 \mathrm{~d}$ ). A recalculation every five 
years seems appropriate given the amount of all new data that can be gathered within that time frame (Pascual et al. 2012).

Incorporating data on beach meiofauna, terrestrial arthropods and vascular plants could permit a more integrative and sound valuation of the coastal zone by addressing the beach ecosystem as a continuum from shallow subtidal waters to the foredunes. However, these ecosystem components are either only scarcely researched or restricted to the foredunes. In the latter case, this would hinder a good relative comparison between all studied zones (foredunes, intertidal and shallow subtidal zones). Limitations on data coverage can be overcome by mapping biophysical characteristics (Young et al. 2007) and subsequent habitat modeling based on, for example, grain size (Van Hoey et al. 2004; Degraer et al. 2008b; Willems et al. 2008), resulting in a sound extrapolation of benthic data to presently unsampled subzones.

Since the marine and coastal environment is very complex, several indicators have been designed to reduce the number of measurements and parameters that normally would be required to give an exact representation of the state of this environment. An indicator in ecology and environmental planning is defined as a component or a measure of environmentally relevant phenomena, e.g. pressures, states and responses, used to depict or evaluate environmental conditions or changes or to set environmental goals (Heink \& Kowarik 2010). Indicators thus require detailed knowledge of what the natural state of a system should be, why the system is in a particular state, and which value-based criteria are necessary for applying the 'good' or 'bad' label (Mee et al. 2008). In general, indicators have to be SMART (specific, measurable, achievable, realistic and time-bounded) such that it will be apparent when they have been met, and when management measures have been successful. In moving towards a more functional approach, the need for indicators of overall health of the system still increases, at the expense of indicators of single aspects of the biota, e.g. species richness and biomass (Borja et al. 2010). Marine biological value is a multi-metric, integrative, system-level ecological indicator developed to be able to assess the intrinsic value of a certain area by integrating all available biological data on different organizational levels of biodiversity (from the species up to the ecosystem level) and for different ecosystem components (Derous et al. 2007d; Borja et al. 2011).

\section{Limitations and Caveats of BV}

The protocol followed in this work reflects the reasoning behind the development of the BV tool, and no fundamental changes to the original assessment questions and concept definitions (Derous 2007) have been undertaken. We highlight that misinterpretations could occur when the BVM is used without consultation of the reliability and availability maps, the underlying maps depicting the results of each assessment question separately per ecosystem component, the documentation of the valuation process or the integrated database. Despite these constraints, the availability of a BVM of the Belgian coast 
allows to answer policy questions related to the biological value of certain subzones in a transparent, objective way, where in the past, managers had to rely mainly on expert judgement (Derous et al. 2007a).

When first applied to the Belgian Part of the North Sea, species richness per subzone was corrected by applying a logistic regression analysis in which besides sampling effort (in terms of area surveyed), the distance to the coast and mean depth were also taken into account (Derous et al. 2007c). However, the BV protocol used here did not yet foresee for such correction, especially since distance to coast and mean depth would be irrelevant factors to be considered in the intertidal and shallow subtidal zone. For future applications, a correction for sampling effort differences among subzones could be designed and applied for questions related to species richness.

The relationship between the spatial coverage of data gathered and the number of subzones established strongly influences the selection for rare species in the BV protocol. Rare species in BV are defined as species appearing in less than $5 \%$ of the studied subzones (Derous et al. 2007d), but this can be changed if properly justified. In this case, all species occur in more than $5 \%$ of the subzones, resulting in a conflict within the selection of rare species. Therefore, rare species were defined as those appearing in less than $10 \%$ of the studied subzones. This can be seen as a rather technical constraint of the protocol and it can be fixed by changing the calculation steps or changing the approach to the selection of rare species (Pascual et al. 2011). Clearly, further attention regarding this matter is fundamental to the successful improvement of the BV protocol.

\section{Conclusions}

The application of the biological valuation framework (Derous et al. 2007a; Derous et al. 2007b) for the shallow Belgian coastal zone was feasible and required minor adjustments. Spatial coverage and overall data availability were satisfactory and allowed for significant trends and patterns to be observed. Although the Belgian coast is entirely composed by sandy beaches, there is indeed biological diversity among distinct subzones and its intrinsic value needs to be properly assessed and taken into account. Spatial information on the intrinsic biological value of a given subzone within areas covered by PSIPS and/or coastal flood risk areas was presented in a straightforward manner, potentially enabling stakeholder's involvement. Similarly, BVMs provided a strong visual support to the proposal for the extension of some already existing nature reserves and to the need for more data to allow for significant conclusions regarding the biological value of other reserves. In both cases however, BVMs should be used along with other criteria defined within a sound decision-support system for spatial planning (Derous et al. 2007c). Important limitations to the applicability of this BV protocol have been identified, mostly related to the threshold for selection of rare species and the approach to calculating species 
Chapter 6-Marine biological valuation of the shallow Belgian coastal zone: a space-use conflict example within the context of marine spatial planning

richness. Notwithstanding these, the highlighted positive aspects strongly suggest that the potentialities of this integrative tool should not be underestimated. Further research on the applications of BV to coastal areas is still required to perfect and fine-tune the tool, enhancing the robustness of its results and consequently strengthening its application within spatial management strategies towards an integrative, ecosystem-based management of coastal areas worldwide. 